G.G. GUNDERSEN AND C.-C. YANG

KODAI MATH. J.

7 (1984), $76-85$

\title{
ON THE PREIMAGE SETS OF ENTIRE FUNCTIONS
}

\author{
By Gary G. Gundersen and Chung-Chun Yang
}

\section{Introduction.}

From the classical Weierstrass factorization theorem we know that for any given sequence $\left\{a_{n}\right\}$ of complex numbers that has no finite limit point, there exists an entire function $f$ such that the sequence $\left\{a_{n}\right\}$ is precisely the zeros of $f$ (counted by multiplicities). A natural question to ask is: given a sequence $\left\{a_{n}\right\}$ that has no finite limit point, does there exist an entire function $f$ such that the sequence $\left\{a_{n}\right\}$ is precisely the zeros and one-points of $f$ ? A similar but more restrictive question was posed by Rubel and Yang [14, p. 289]. The answer to the question here, in general, is no.

Gross [4] made the following general definition.

DEFINITION. A countable discrete set $\Omega$ is defined to be a nontrivial preimage set (NPS) if there exists a nonlinear entire function $f$ and a set $S$ of distinct complex values with $2 \leqq|S|<\infty \quad(|S|$ denotes the cardinality of $S)$ such that $f^{-1}(S)=\Omega$, where multiplicities are counted accordingly (thus the elements in $\Omega$ need not be distinct).

The problem arises to try to completely characterize the set of entire functions $\{f\}$ associated with an NPS $\Omega$. Gross and Yang [7] exhibited some nontrivial preimage sets $\{\Omega\}$ that are unique in the sense that if $\Omega=f^{-1}\left(S_{1}\right)=g^{-1}\left(S_{2}\right)$ for entire functions $f, g$ and corresponding finite sets $S_{1}, S_{2}$, then $f \equiv a g+b$ for constants $a \neq 0, b$. We will prove the following result which completely characterizes the set of entire functions associated with a "simple periodic" NPS $\Omega=\{b, b \pm a, b \pm 2 a, \cdots\}$ where $a \neq 0$ and $b$ are constants.

THEOREM 1. If $f$ is an entire function for the NPS $\Omega=\{0, \pm \pi, \pm 2 \pi, \cdots\}$, then $f$ necessarily has one of the following forms (up to a linear transformation):

1. $f(z)=\exp \left(\frac{2 i z}{m}\right)$ or $f(z)=\exp \left(-\frac{2 i z}{m}\right)$ where $m$ is a positive integer.

2. $f(z)=\sin \left(\frac{z}{n}+\frac{\pi}{2 n}+\frac{m \pi}{n}\right)$ where $n$ is an even integer $\geqq 2$ and $m$ is an integer.

3. $f(z)=\sin \left(\frac{z}{n}+\frac{m \pi}{n}\right)$ where $n$ is an odd integer $\geqq 3$ and $m$ is an integer.

Received March 22, 1983 
We note that $\exp ( \pm 2 i z)-1=\left[\exp \left( \pm \frac{2 i z}{m+1}\right)\right]^{m+1}-1$ and

$[\exp ( \pm 2 i z)-1] \exp \left( \pm \frac{2 i z}{m}\right)=\left\{\left[\exp \left( \pm \frac{2 i z}{m}\right)\right]^{m}-1\right\} \exp \left( \pm \frac{2 i z}{m}\right) ;$ thus $\Omega$ is an NPS for each function $f$ in case 1 of Theorem 1 . It can be deduced from the proof of Theorem 1 that these are essentially the only situations that can occur in case $1 . \quad \Omega$ is also an NPS for each function $f$ in cases 2 and 3 from the wellknown multiple angle formulas; specifically, for each such $f$ we can find a polynomial $P(z)$ so that $\sin z \equiv P(f(z))$, and $P$ is necessarily unique and of degree $n$. Thus Theorem 1 is an optimal result.

Two of the open questions that were posed in [4], [7] are:

1. For any given NPS $\Omega$, does there exist some finite nonempty set $\Omega_{0}$ (i.e., $\left.1 \leqq\left|\Omega_{0}\right|<\infty\right)$ so that $\Omega \cup \Omega_{0}$ or $\Omega-\Omega_{0}$ is also an NPS ? (in the case $\Omega-\Omega_{0}$ it is required that $\Omega_{0} \subset \Omega$ ).

2. Does there exist an infinite NPS $\Omega$ such that for any finite nonempty set $\Omega_{0}, \Omega \cup \Omega_{0}$ and $\Omega-\Omega_{0}$ are nontrivial preimage sets?

The answer to question 1 is no by the following result.

THEOREM 2. Given the NPS $\Omega=\{b, b \pm a, b \pm 2 a, \cdots\}$ where $a \neq 0$ and $b$ are constants. Suppose that we either $(A)$ add a finite set of points to $\Omega$, or $(B)$ remove a finite set of points from $\Omega$. Then the resulting set will not be an NPS.

Examples 1 and 2 in $\S 3$ show that there exist nontrivial preimage sets that will remain nontrivial preimage sets after we add or remove some particular finite set of points. We suspect that the answer to question 2 is no also.

In this paper we will assume that the reader is familiar with the standard notations and fundamental results of Nevanlinna's theory of meromorphic functions [9], [10].

In $\S 2$ we will prove Theorem 1, plus make some remarks and pose some questions about functions associated with the same NPS.

In $\S 3$ we will prove Theorem 2, give the above-mentioned Examples 1 and 2 , and pose some questions about when an NPS is "changed slightly".

In $\S 4$ we will exhibit several classes of countable discrete sets that are not nontrivial preimage sets.

We would like to thank the referee for an observation that made an improvement in this paper.

\section{The functions associated with the same NPS.}

We will now prove Theorem 1.

LEMMA 1. If $\Omega$ is an NPS for the entire function $f$, then the order of $f$ is equal to the exponent of convergence of $\Omega$. 
Lemma 1 follows immediately from the classical theorem of Borel.

Proof of Theorem 1. By combining the hypothesis of Theorem 1 with Lemma 1 we obtain that

$$
e^{c z}\left(e^{2 i z}-1\right) \equiv p(f(z))
$$

where $c$ is some constant and $p(z)=A\left(z-a_{1}\right) \cdots\left(z-a_{n}\right)$ is a polynomial with $n \geqq 2$ distinct zeros $a_{1}, \cdots, a_{n}$.

From a result of Ritt [13] it follows from (1) that $f$ is necessarily an exponential polynomial; that is

$$
f(z)=A_{0}+\sum_{k=1}^{m} A_{k} \exp \left(B_{k} z\right)
$$

where the $A_{k}$ 's are constants $\left(A_{k} \neq 0\right.$ for $\left.1 \leqq k \leqq m\right)$ and the $B_{k}$ 's are distinct nonzero constants.

Suppose $m=1$. Then from (1), (2), and the Borel identity theorem [10, p. 113] it follows that we have one of the following four cases: $c=0$ and $n B_{1}=2 i$, $c=-2 i$ and $n B_{1}=-2 i, c=B_{1}$ and $(n-1) B_{1}=2 i$, or $c=B_{1}-2 i$ and $(n-1) B_{1}=-2 i$. This is case 1 in Theorem 1 .

Now suppose $m \geqq 2$. Set $B_{k}=\lambda_{k}+i \mu_{k}$. We can assume that $B_{1}$ and $B_{2}$ are the two particular constants among the $B_{k}$ 's that satisfy $\lambda_{1}=\max \left\{\lambda_{k}\right\}, \mu_{1}=$ $\max \left\{\mu_{k}: \lambda_{k}=\lambda_{1}\right\}$, and $\lambda_{2}=\min \left\{\lambda_{k}\right\}, \mu_{2}=\min \left\{\mu_{k}: \lambda_{k}=\lambda_{2}\right\}$. From (1), (2), and the Borel identity theorem it follows that either $n B_{1}=c+2 i$ and $n B_{2}=c$, or $n B_{1}=c$ and $n B_{2}=c+2 i$. Hence $\lambda_{1}=\lambda_{2}$. With similar reasoning we can now deduce that $m=2$ in (2). It can then be seen (with similar reasoning) that $(n-1) B_{1}+B_{2}$ must equal either $c+2 i, c$, or $m B_{1}+k B_{2}$ where $m \neq n-1$ and $k \neq 1$. Thus $(n-1) B_{1}+B_{2}$ $=m B_{1}+k B_{2}$. Since $n\left(B_{1}-B_{2}\right)= \pm 2 i$, we obtain $\lambda_{1}=\lambda_{2}=0$. Hence $c=\beta i$ where $\beta$ is real $(\beta \neq 0,-2)$.

Now we differentiate (1) twice and eliminate $\exp ((c+2 i) z)$ and $\exp (c z)$ between the three equations to obtain

$$
2 i(\beta+1) p^{\prime}(f) f^{\prime}-p^{\prime \prime}(f)\left(f^{\prime}\right)^{2}-p^{\prime}(f) f^{\prime \prime}+\beta(\beta+2) p(f) \equiv 0 .
$$

From Nevanlinna's second fundamental theorem [9, pp. 43-44] it follows that we can find distinct points $b_{1}, \cdots, b_{n-1}$, and one sequence $r_{3} \rightarrow+\infty$ such that

$$
\sum_{k=1}^{n-1} m\left(r_{j}, f, b_{k}\right)=o(1) T\left(r_{j}, f\right) \quad \text { as } \quad r_{j} \rightarrow+\infty \text {. }
$$

For a given $a_{k}$ and $q(f)=\left(f-b_{1}\right) \cdots\left(f-b_{n-1}\right)$ we have from (3),

$$
\frac{\beta(\beta+2) p(f)}{q(f)\left(f-a_{k}\right)} \equiv \frac{p^{\prime \prime}(f)\left(f^{\prime}\right)^{2}}{q(f)\left(f-a_{k}\right)}+\frac{p^{\prime}(f) f^{\prime \prime}}{q(f)\left(f-a_{k}\right)}-\frac{2 i(\beta+1) p^{\prime}(f) f^{\prime}}{q(f)\left(f-a_{k}\right)} .
$$

By using the partial fraction decomposition of each term in (5) together with (4) and Nevanlinna's fundamental estimate of the logarithmic derivative, we can deduce that $m\left(r_{j}, f, a_{k}\right)=o(1) T\left(r_{j}, f\right)$ as $r_{j} \rightarrow \infty$. Hence 


$$
m\left(r_{j}, f, a_{k}\right)=o(1) T\left(r_{j}, f\right) \quad \text { for } \quad 1 \leqq k \leqq n .
$$

Now from (1), (6), and [15, Satz 1] we have as $r=r_{j} \rightarrow \infty$,

$$
\begin{aligned}
\frac{2 r}{\pi}(1+o(1)) & =N\left(r, e^{(\beta+2) i z}-e^{\beta i z}, 0\right)=\sum_{k=1}^{n} N\left(r, f, a_{k}\right) \\
& =(n+o(1)) T(r, f) \geqq T\left(r, e^{(\beta+2) i z}-e^{\beta i z}\right) \\
& = \begin{cases}(\beta+2+o(1)) \frac{r}{\pi} & \text { if } \quad \beta>0, \\
-(\beta+o(1)) \frac{r}{\pi} & \text { if } \quad \beta<-2, \\
(1+o(1)) \frac{2 r}{\pi} & \text { if }-2<\beta<0 .\end{cases}
\end{aligned}
$$

Hence $-2<\beta<0$ is necessary.

Now we can use elementary reasoning with (1) and (2) to deduce that $\beta=-1$ and

$$
f(z)=A_{0}+A_{1} \exp \left(\frac{i z}{n}\right)+A_{2} \exp \left(-\frac{i z}{n}\right) .
$$

Further elementary analysis of (7) and (1) will yield cases 2 and 3 in Theorem 1. The proof of Theorem 1 is now complete.

Open questions.

1. Does the analogous result to Theorem 1 hold for the NPS $\Omega=\{z: \exp (h(z))=1\}$ where $h$ is any nonconstant entire function? (Note: In this paper a set in the form $\{z: F(z)=0\}$ will always count multiplicities.)

2. Does there exist an uncountable family of entire functions $\left\{f_{i}\right\}$ associated with some NPS $\Omega$ such that $f_{i} \not \equiv a f_{j}+b$ if $i \neq j$ ? We can have a countably infinite family from Theorem 1.

Remark 1. Concerning the topic of an NPS for two entire functions $f$ and $g$ (where $f \not \equiv a g+b$ ) we mention the following studies.

(i) The papers of Gross [2] and Gross and Yang [6] contain a study of some special cases of entire functions that are associated with the same NPS.

(ii) We say that two nonconstant entire functions $f$ and $g$ share the finite value $a \mathbf{C M}$ when $f(z)-a$ and $g(z)-a$ have the same zeros counting multiplicities. Thus two distinct nonconstant entire functions that share two or three finite values CM will give an example of an NPS for two entire functions that are not linear transformations of each other. The form of two such entire functions was given by Nevanlinna [10, pp. 122-125]. Two nonconstant entire functions that share four finite values (ignoring multiplicities) must be identical from the well-known theorem of Nevanlinna [10, p. 109]. 
Remark 2. Theorem 5.11 in [3] is a result in the general area of Theorem 1 .

\section{Adding (removing) a finite set to (from) an NPS.}

Proof of Theorem 2. Let $R(z)$ be a nonconstant rational function such that $R(z) \sin z$ is entire and where $R(z) \rightarrow \infty$ or $R(z) \rightarrow 0$ as $z \rightarrow \infty$. We will show that the set $\Omega=\{z: R(z) \sin z=0\}$ is not an NPS, and this will prove Theorem 2 .

Suppose $\Omega$ is an NPS. Then from Lemma 1 we have

$$
R(z) e^{c z} \sin z \equiv p(f(z))
$$

where $c$ is a constant and $p$ is a nonlinear polynomial with distinct zeros $a_{1}, \cdots, a_{n}$.

Suppose that $p^{\prime}(f(z))=0$ has only a finite number of roots. It then follows from (8) and Picard's theorem that $R(z) e^{c z} \sin z \equiv B+\left(q(z) e^{A z}\right)^{n}$ where $q$ is a polynomial and $A, B$ are constants. Logarithmic differentiation of this identity yields

$$
\frac{R^{\prime}(z)}{R(z)}+c+\cot z \equiv \frac{n(q(z))^{n-1}\left(q^{\prime}(z)+A q(z)\right) e^{n A z}}{B+(q(z))^{n} e^{n A z}} .
$$

Since $\frac{R^{\prime}}{R}+c$ is nonconstant and $\cot z$ has the two Picard values $\pm i$, it easily follows from Nevanlinna's three-functions theorem [9, p. 47] that $\frac{R^{\prime}}{R}+c+\cot z$ has an infinite number of zeros, which contradicts (9). Hence there must exist a constant $b$ such that $p^{\prime}(b)=0$ and the equation $f(z)=b$ has an infinite number of roots $\left\{z_{m}\right\}_{m=1}^{\infty}$.

Set $B=p(b)$. Since $R(z) \sin z$ can have at most a finite number of multiple zeros, it follows from (8) that $B$ is nonzero. Then from (8), each $z=z_{m}$ satisfies the following two equations:

$$
\begin{gathered}
R(z) e^{c z} \sin z=B ; \\
\frac{R^{\prime}(z)}{R(z)} B+c B+R(z) e^{c z} \cos z=0 .
\end{gathered}
$$

From (10) and (11) we obtain

$$
e^{2 c z}=\left(\frac{B}{R(z)}\right)^{2}\left[1+\left(\frac{R^{\prime}(z)}{R(z)}+c\right)^{2}\right] .
$$

Combining (12) and (10) yields

$$
\sin ^{2} z=\frac{1}{1+\left(\frac{R^{\prime}(z)}{R(z)}+c\right)^{2}} .
$$

Suppose first that $c \neq \pm i$. Then as $z_{m} \rightarrow \infty$, 


$$
\sin ^{2}\left(z_{m}\right) \rightarrow \frac{1}{1+c^{2}} .
$$

If $z_{m}=x_{m}+i y_{m}$ then for some constant $M \geqq 0$,

$$
\left|y_{m}\right| \leqq M \quad \text { for all } m \text {. }
$$

An elementary analysis of (15), (14), and (10) combined with the hypothesis on $R$ will show that either $B=0$ or $B=\infty$. Thus we have a contradiction.

Now suppose that $c=i$. Then from (12) and (10) we obtain

$$
\frac{B^{2}}{R\left(z_{m}\right)}\left[\left(\frac{R^{\prime}\left(z_{m}\right)}{R\left(z_{m}\right)}\right)^{2}+2 i \frac{R^{\prime}\left(z_{m}\right)}{R\left(z_{m}\right)}\right]=R\left(z_{m}\right)+2 i B .
$$

Since $R$ is rational it follows that

$$
B^{2}\left[\left(R^{\prime}(z)\right)^{2}+2 i R^{\prime}(z) R(z)\right] \equiv(R(z)+2 i B)(R(z))^{3} .
$$

From (16) we see that $R$ can have no zeros. Thus $R(z) \rightarrow 0$ as $z \rightarrow \infty$. But then from (10), $R\left(z_{m}\right) \exp \left(2 i z_{m}\right) \rightarrow 2 i B$, while from (11), $R\left(z_{m}\right) \exp \left(2 i z_{m}\right) \rightarrow-2 i B$, thus producing the contradiction $B=0$. Hence $c=i$ is impossible. We can deduce that $c=-i$ is also impossible.

We have a contradiction in all cases, and so the proof of Theorem 2 is complete.

In contrast with Theorem 2 the next two examples show that for some nontrivial preimage sets it is possible to add (remove) some particular finite set of points and have the resulting set remain an NPS.

EXAMPLE 1. Let $q$ be a nonconstant polynomial, $h$ a nonconstant entire function, and $p$ a nonlinear polynomial with distinct zeros. Then $\Omega=$ $\left\{z: p\left(q(z) e^{h(z)}\right)=0\right\}$ is obviously an NPS for $q e^{h}$.

Suppose first that $p(0) \neq 0$. Then $\Omega_{0}=\{z: q(z)=0\}$ is a finite nonempty set and

$$
\Omega \cup \Omega_{0}=\left\{z: q(z) e^{h(z)} p\left(q(z) e^{h(z)}\right)=0\right\}
$$

is clearly an NPS for $q e^{h}$.

Now suppose that $p(0)=0$ and $\operatorname{deg}(p) \geqq 3$. Then

$$
\Omega-\Omega_{0}=\left\{z: \frac{p\left(q(z) e^{h(z)}\right)}{q(z) e^{h(z)}}=0\right\}
$$

is clearly an NPS for $q e^{h}$.

EXAMPlE 2. Let $2 \alpha$ be a positive integer. We will show that there exists an NPS $\Omega$ with exponent of convergence $\alpha$ with the following property: $\Omega$ is exactly the zeros and ones of some entire function $g$ and there exists a particular finite nonempty set $\Omega_{0}$ such that $\Omega \cup \Omega_{0}$ is exactly the zeros and ones of another entire function $f$. Obviously then the same statement holds if " $\Omega_{0}$ such that 
$\Omega \cup \Omega_{0}$ " is replaced by " $\Omega_{0} \subset \Omega$ such that $\Omega-\Omega_{0}$ ".

To prove this assertion we consider a functional equation of the form

$$
f(f-1)=p g(g-1)
$$

where $p$ is any nonconstant polynomial such that $\operatorname{deg}(p)=2 \alpha$, and where $f$ and $g$ are required to be entire functions. Equation (17) can be written as

$$
F^{2}-p G^{2}=\frac{1}{4}-\frac{p}{4}
$$

where $F=f-1 / 2$ and $G=g-1 / 2$. From theorems of Gross, Osgood, and Yang [5; Theorems I, III, V] it follows that there exists a pair of entire functions $F$ and $G$ that are a solution of (18) such that $\operatorname{order}(F)=\operatorname{order}(G)=\alpha$. Hence there exist entire functions $f$ and $g$ that satisfy (17) with $\operatorname{order}(f)=\operatorname{order}(g)=\alpha$. Thus in view of (17) the assertion is proved.

Open questions.

1. If, in the hypothesis of Theorem 2, we replace the set $\Omega$ with the set $\Omega=$ $\{z: \exp (h(z))=1\}$ where $h$ is nonconstant entire, will the conclusion of Theorem 2 still hold? We suspect the answer is yes.

2. Let $\Omega$ be an NPS that has a finite exponent of convergence that is not onehalf of an integer. Is it true that if we add (remove) a finite set of points to (from) $\Omega$ then the resulting set will no longer be an NPS ?

3. Suppose that $\Omega=\left\{c_{n}\right\}$ is an infinite NPS. Does there exist an infinite sequence of positive numbers $\left\{\delta_{n}\right\}$ where $\delta_{n} \rightarrow 0$ with the following property: if $\Omega^{\prime}=\left\{d_{n}\right\}$ is any infinite discrete set that satisfies $0<\left|c_{n}-d_{n}\right|<\delta_{n}$ for all $n$ then $\Omega^{\prime}$ is not an NPS ?

A special case of an NPS is a zero-one set [14], which is a pair of sequences $\left(\left\{a_{n}\right\},\left\{b_{n}\right\}\right)$ without finite limit points such that there is an entire function $f$ whose zero-sequence is precisely $\left\{a_{n}\right\}$ and whose one-sequence is precisely $\left\{b_{n}\right\}$ (counted by multiplicities). Some papers on zero-one sets are [11], [12], [14], and [16]. Open question 3 above was studied by Winkler [16] for zero-one sets. See also [11].

\section{Some sets that are not nontrivial preimage sets.}

Theorem 2 gives a class of countable discrete sets that are not non-trivial preimage sets. We will exhibit here several more classes.

EXAMPLE 3. Let $\Omega$ be a countable discrete set of real numbers with an exponent of convergence $\lambda>1$. It is easy to see that $\Omega$ cannot be an NPS because of Edrei's theorem [1] that an entire function with only real zeros and real ones has order at most one. Miles [14, p. 289] used this reasoning to prove the analogous result concerning zero-one sets. 
EXAMPLE 4 . Let $\Omega$ be a countable discrete nonempty set such that every point in $\Omega$ appears at least three times, and let $\Omega_{1}$ be a countable discrete set that satisfies

$$
\lim _{r \rightarrow \infty} \frac{\bar{N}\left(r, \Omega_{1}\right)}{N(r, \Omega)}=0
$$

Then $\Omega \cup \Omega_{1}$ is not an NPS. The proof of this result is similar to the proof of Theorem 1 in [7], and hence we will omit it. This result generalizes Theorem 1 of $[7]$.

THEOREM 3. Let $F$ be an entire function that either $(A)$ has finite noninteger order $\rho$, or $(B)$ has integer order $\rho \geqq 1$, maximum type, and the exponent of convergence of the zeros of $F$ is $\rho$. Suppose that for $0 \leqq \lambda<1$,

$$
\bar{N}(r, F, 0) \leqq(\lambda+o(1)) T(r, F)
$$

as $r \rightarrow \infty$. Set $\Omega=\{z: F(z)=0\}$.

If $0 \leqq \lambda<1 / 2$ then $\Omega$ is not an NPS. In the other cases $1 / 2 \leqq \lambda<1$, suppose that $\Omega$ is an NPS for some entire function $f$; that is

$$
F e^{h}=\left(f-a_{1}\right)\left(f-a_{2}\right) \cdots\left(f-a_{n}\right)
$$

where $h$ is entire anp $a_{1}, \cdots, a_{n}$ are $n \geqq 2$ distinct numbers. Then

$$
\lambda \geqq \frac{n-1}{n} \text {. }
$$

Proof. Suppose $\Omega$ is an NPS, i.e. (20) is satisfied. From Lemma 1 and the hypothesis we see that in (20), order $(f)=\rho$ and $h$ is a polynomial of degree $\leqq \rho$. It follows that there exists a sequence $r_{m} \rightarrow+\infty$ such that $T\left(r_{m}, e^{-h}\right)=$ $o(1) T\left(r_{m}, F\right)$. Then from (20) and the second fundamental theorem we obtain

$$
\begin{aligned}
\bar{N}\left(r_{m}, 0, F\right) & =\sum_{k=1}^{n} \bar{N}\left(r_{m}, a_{k}, f\right) \geqq(n-1+o(1)) T\left(r_{m}, f\right) \\
& \geqq\left(\frac{n-1}{n}+o(1)\right) T\left(r_{m}, F\right) .
\end{aligned}
$$

Combining this with (19) will prove Theorem 3.

Remarks.

1. If $F$ has integer order and normal type then we cannot get an analogous result to Theorem 3 ; consider for example

$$
F(z)=e^{c z}\left(e^{z}-a_{1}\right)\left(e^{z}-a_{2}\right) \cdots\left(e^{z}-a_{n}\right) .
$$

2. It is possible to get equality in (21) for every $n$ in case (A) of Theorem 3 ; consider the function 


$$
F(z)=(\cos \sqrt{z}-1)(\cos \sqrt{z}+1)(\cos \sqrt{z}+2) \cdots(\cos \sqrt{z}+n-1) .
$$

THEOREM 4. Suppose $F$ is entire of finite order $\rho>0$, the zeros of $F$ have exponent of convergence $\rho$, and $\bar{N}(r, F, 0)=o(1) r^{\rho-\varepsilon}$ for some $\varepsilon>0$. Then $\Omega=$ $\{z: F(z)=0\}$ is not an NPS.

Proof. If $\Omega$ is an NPS, then

$$
F e^{h}=\left(f-a_{1}\right) \cdots\left(f-a_{n}\right)
$$

where $h$ is entire, $f$ is entire of order $\rho$ from Lemma 1 , and $a_{1}, a_{2}, \cdots, a_{n}$ are $n \geqq 2$ distinct values. Hence by the second fundamental theorem,

$$
(n-1+o(1)) T(r, f) \leqq \sum_{k=1}^{n} \bar{N}\left(r, f, a_{k}\right)=\bar{N}(r, F, 0)=o(1) r^{\rho-\varepsilon},
$$

which is a contradiction. This proves Theorem 4 .

Remark. The zeros of a function $F$ in Theorem 4 will necessarily have unbounded multiplicities. An example [8, pp. 298-299] is

$$
F(z)=\exp \left[\sum_{n=0}^{2 m} a_{n}(2 \pi i)^{-n} \int \frac{p^{\prime}(z)(p(z))^{n}}{e^{p(z)}-1} d z\right],
$$

where $m$ is a positive integer, $p$ is a nonconstant polynomial, and $\left\{a_{n}\right\}_{n=0}^{2 m}$ are rational numbers $\left(a_{2 m}>0\right)$ such that $\sum_{n=0}^{2 m} a_{n} k^{n}$ is a non-negative integer whenever $k$ is an integer. $F$ is entire of $\operatorname{order}(2 m+1) \operatorname{deg}(p)$, the exponent of convergence of the zeros of $F$ is $(2 m+1) \operatorname{deg}(p)$, and $\bar{N}(r, F, 0)=\bar{N}\left(r, e^{p}, 1\right)+\mathrm{O}(\log r)$.

Of course we can easily construct canonical products $F$ to satisfy the hypothesis of Theorem 4.

\section{REFERENCES}

[1] A. EDREI, Meromorphic functions with three radially distributed values, Trans. Amer. Math. Soc., 78 (1955), 276-293.

[2] F. Gross, On the distribution of values of meromorphic functions, Trans. Amer. Math. Soc., 131 (1968), 199-214.

[3] F. Gross, Factorization of meromorphic functions, U.S. Gov. Printing Office, 1972.

[4] F. Gross, Factorization of meromorphic functions and some open problems, in Complex analysis, Kentucky 1976 (Proc. Conf.), Lecture notes in mathematics, no. 599, pp. 51-67, Springer-Verlag, Berlin/Heidelberg/New York, 1977.

[5] F. Gross, C.F. Osgood AND C.C. YANG, On the entire solutions of a functional equation in the theory of fluids, J. Math. Phys. 16 (1975), 2142-2147.

[6] F. GRoss AND C.C. YANG, Meromorphic functions covering certain finite sets at the same points, Illinois J. Math., 26 (1982), 432-441.

[7] F. Gross AND C.C. YANG, On preimage and range sets of meromorphic functions, Proc. Japan Acad., 58 Ser. A (1982), 17-20. 
[8] G. G. Gundersen, When two entire functions and also their first derivatives have the same zeros, Indiana Univ. Math. J., 30 (1981), 293-303.

[9] W. K. HAYMan, Meromorphic functions (Clarendon Press, Oxford, 1964).

[10] R. Nevanlinna, Le théorème de Picard-Borel et la théorie des fonctions méromorphes (Gauthier-Villars, Paris, 1929).

[11] M. Ozawa, On the zero-one set of an entire function, Kōdai Math. Sem. Rep., 28 (1977), 311-316.

[12] M. Ozawa, On the zero-one set of an entire function, II, Kodai Math. J., 2 (1979), 194-199.

[13] J.F. Ritr, Algebraic combinations of exponentials, Trans. Amer. Math. Soc., 31 (1929), 654-679.

[14] L. A. RUBEL AND C.C. YANG, Interpolation and unavoidable families of meromorphic functions, Michigan Math. J., 20 (1973), 289-296.

[15] N. Steinmetz, Zur wertverteilung von exponentialpolynomen, manuscripta math., 26 (1978), 155-167.

[16] J. WINKLER, Zur existenz ganzer funktionen bei vorgegebener menge der nullstellen und einsstellen, Math. Z., 168 (1979), 77-85.

Gary G. Gundersen

Department of Mathematics

UNIVERSity OF NEW ORLEANS

NEW ORLEANS, LA 70148

U.S. A.
Chung-Chun Yang

Naval Research Laboratory

Washington, D. C. 20375

U.S. A. 